\title{
Modeling Seasonal Fractionally Integrated Autoregressive Moving Average-Generalized Autoregressive Conditional Heteroscedasticity Model with Seasonal Level Shift Intervention
}

\author{
Lawrence Dhliwayo', Florance Matarise' ${ }^{1}$, Charles Chimedza² \\ ${ }^{1}$ Department of Statistics, University of Zimbabwe, Harare, Zimbabwe \\ ${ }^{2}$ School of Statistics and Actuarial Science, University of Witwatersrand, Johannesburg, South Africa \\ Email: larry7dhl@gmail.com, matarise7@gmail.com, ccvisuals@gmail.com
}

How to cite this paper: Dhliwayo, L., Matarise, F. and Chimedza, C. (2020) Modeling Seasonal Fractionally Integrated Autoregressive Moving Average-Generalized Autoregressive Conditional Heteroscedasticity Model with Seasonal Level Shift Intervention. Open Journal of Statistics, 10, 810-831.

https://doi.org/10.4236/ojs.2020.105047

Received: August 19, 2020

Accepted: October 24, 2020

Published: October 27, 2020

Copyright $\odot 2020$ by author(s) and Scientific Research Publishing Inc. This work is licensed under the Creative Commons Attribution International License (CC BY 4.0).

http://creativecommons.org/licenses/by/4.0/

\begin{abstract}
This paper introduces the class of seasonal fractionally integrated autoregressive moving average-generalized conditional heteroskedastisticty (SARFIMAGARCH) models, with level shift type intervention that are capable of capturing simultaneously four key features of time series: seasonality, long range dependence, volatility and level shift. The main focus is on modeling seasonal level shift (SLS) in fractionally integrated and volatile processes. A natural extension of the seasonal level shift detection test of the mean for a realization of time series satisfying SLS-SARFIMA and SLS-GARCH models was derived. Test statistics that are useful to examine if seasonal level shift in an SARFIMA-GARCH model is statistically plausible were established. Estimation of SLS-SARFIMA and SLS-GARCH parameters was also considered.
\end{abstract}

\section{Keywords}

Seasonality, Fractional Integration, Long-Memory, Level Shift, SLS-SARFIMA, SLS-GARCH, Volatility

\section{Introduction}

The phenomenon of long memory or long range dependence in time series processes has been of interest in time series research. A popular way to analyze a long memory time series is to use seasonal autoregressive fractionally integrated moving average (SARFIMA) processes introduced by [1] and [2]. The works of [1] and [2] assume that the conditional variance of the time series is constant over time. However, non constant variance in non-linear time series is a chal- 
lenging modelling exercise, considered among other things by [3]. In particular, the stylized fact that the volatility of financial time series is non constant has been long recognized in literature, see for example [4] [5] and [6].

The methodology for modelling time series with long memory behavior has been extended to long memory time series with time varying conditional variance, see for instance, [7] who developed the ARFIMA model with generalized autoregressive conditional heteroskedasticity (GARCH) type innovations, and [8] examine the daily average $\mathrm{PM}_{10}$ concentration using a seasonal ARFIMA model with GARCH errors. Tong [9] analyzed the nonlinear time series using GARCH models and [10] used GARCH models for testing market efficiency. These models do not capture level shifts both in mean and variance, in this paper we introduce a new class of SARFIMA-GARCH models with seasonal level shift intervention in mean and volatility. This approach allows us to model mean and volatility seasonal level shifts in an SARFIMA-GARCH model which is often observed in financial or economics time series.

This article introduces detection of a mean and volatility level shifts innovation in an ARFIMA-GARCH model. The works of [11] first applied ARFIMA-GARCH models to price indices then [7] derived conditions for asymptotic normality of the approximate (Gaussian) maximum likelihood (ML) estimator in the ARFIMA-GARCH model. This paper also extends parameter estimation for an SARFIMA-GARCH model to case with seasonal level shift which we will denote Seasonal Level Shift SARFIMA (SLS-ARFIMA) and Seasonal Level Shift GARCH (SLS-GARCH) using quasi-maximum likelihood estimation.

The first concern of this paper is how one would formally address modeling mean and volatility seasonal level shifts in an SARFIMA-GARCH. The second concern is derivation of test statistics that are useful to examine presence of seasonal level shifts in mean and volatility for an SARFIMA-GARCH model. The layout of the paper is organised as follows. Section 2 reviews some theoretical results of SARFIMA-GARCH and intervention. In Section 3, we introduce the class of SLS-ARFIMA-SLS-GARCH models. Section 4 deals with parameter estimation in SLS-ARFIMA and SLS-GARCH models. Section 5 is dedicated to the proposed procedure of level shift detection in SARFIMA-GARCH models. The last section concludes with the main findings and limitations. Common acronyms used in this paper are given in Table 1.

Table 1. Common acronyms used in this paper.

\begin{tabular}{|c|c|}
\hline Acronym & Explanation \\
\hline $\mathrm{AO}$ & Additive outlier \\
\hline LS & Level shift \\
\hline $\mathrm{TC}$ & Temporary change \\
\hline IO & Innovative outlier \\
\hline SLS & Seasonal level shift \\
\hline ARMA & Autoregressive moving average \\
\hline
\end{tabular}


Continued

\begin{tabular}{cl}
\hline ARFIMA & Autoregressive fractionally integrated moving average \\
SARFIMA & Seasonal autoregressive fractionally integrated moving average \\
GARCH & Generalized autoregressive conditional heteroskedasticity \\
LS-ARFIMA & Level shift-autoregressive fractionally integrated moving average \\
LS-GARCH & Level shift-generalized autoregressive conditional heteroskedasticity \\
SLS-ARFIMA & Seasonal level shift-autoregressive fractionally integrated moving average \\
SLS-GARCH & Seasonal level shift-generalized autoregressive conditional heteroskedasticity \\
\hline
\end{tabular}

\section{SARFIMA-GARCH Models}

This section presents review of the ARFIMA models, SARFIMA models, GARCH models and the SARFIMA-GARCH models. The variance of GARCH model and intervention in ARFIMA models is also presented.

\subsection{ARFIMA Process}

Time series analysis has turned attention to the studies with long memory or long-range dependence characteristics. The $\operatorname{ARFIMA}(p, d, q)$ process, first introduced by [1] and [2], present this property when the differencing parameter $d$ is in the interval $(0,0.5)$. This feature is reflected by the hyperbolic decay of its autocorrelation function or by the unboundedness of its spectral density function, while in the ARMA model, dependency between observations decays at a geometric rate.

Montanari et al. [12] introduced a special form of the generalized ARFIMA model considered by [13]. This formulation is able to reproduce short-and long memory periodicity in the autocorrelation function of the process. Using the [14] notation, the general form of the ARFIMA model is defined as follows:

Let $\left\{x_{t}\right\}_{t \in \mathbb{Z}}$ be a stochastic process, then $\left\{x_{t}\right\}_{t \in \mathbb{Z}}$ is a zero mean $\operatorname{ARFIMA}(p, d, q)$ process given by the expression

$$
\phi(B)(1-B)^{d} x_{t}=\theta(B) \varepsilon_{t}, \text { for } t \in \mathbb{Z}
$$

where $\left\{\varepsilon_{t}\right\}_{t \in \mathbb{Z}}$ is a white noise process with zero mean and variance $\sigma_{\varepsilon}^{2}=\mathbb{E}\left(\varepsilon_{t}^{2}\right)$, $B$ is the backward-shift operator, that is, $B^{k} x_{t}=x_{t-k}$ and $(1-B)^{d}$ is the non seasonal difference, $\phi(\cdot)$ and $\theta(\cdot)$ are the non-seasonal polynomials of degrees $p$ and $q$, respectively, defined by:

$$
\phi(B)=\sum_{i=0}^{p}\left(-\phi_{i}\right) B^{i}, \theta(B)=\sum_{j=0}^{q}\left(-\theta_{j}\right) B^{j},
$$

where $\phi_{i}, 1 \leq i \leq p$, and $\theta_{j}, 1 \leq j \leq q$ are constants and $\phi_{0}=-1=\theta_{0}$.

The difference operator $(1-B)^{d}$ is defined by means of the binomial expansion:

$$
(1-B)^{d}=\sum_{j=0}^{\infty}\left(\begin{array}{l}
d \\
j
\end{array}\right)(-B)^{j}
$$

where; 
$\left(\begin{array}{l}d \\ j\end{array}\right)=\frac{\Gamma(1+d)}{\Gamma(1+j) \Gamma(1+d-j)}$ and $\Gamma(\cdot)$ is the well known gamma function.

The ARFIMA model is said to be stationary when $-0.5<d<0.5$. The model becomes nonstationary when $d \geq 0.5$ and stationary but non invertible when $d \leq-0.5$. The ARFIMA model represents a short memory if $d=0$ and a unit root process is shown when $d=1$. Furthermore, the model has a positive dependence among distance observations or the so called long memory process if $0<d<0.5$; and it also has an anti-persistent property or has an intermediate memory if $-0.5<d<0$.

\subsection{SARFIMA $(p, d, q) \times(P, D, Q) s$ Process}

The seasonal autoregressive fractionally integrated moving average process, denoted hereafter by $\operatorname{SARFIMA}(p, d, q) \times(P, D, Q) s$, is an extension of the long range dependence in the mean $\operatorname{ARFIMA}(p, d, q)$ process, proposed by [1] and [2]. The $\operatorname{SARFIMA}(p, d, q) \times(P, D, Q) s$ process describes time series with long memory or long range dependence or persistent periodical behavior at finite number of spectrum frequencies.

A special form of the generalized ARFIMA model was considered by [13]. This formulation is able to reproduce short and long memory periodicity in the autocorrelation function of the process. Using the [14] notation, the general form of the SARFIMA model is defined below:

Let $\left\{x_{t}\right\}_{t \in \mathbb{Z}}$ be a stochastic process, then $\left\{x_{t}\right\}_{t \in \mathbb{Z}}$ is a zero mean $\operatorname{SARFIMA}(p, d, q) \times(P, D, Q) s$ process given by the expression

$$
\phi(B) \Phi\left(B^{s}\right)(1-B)^{d}\left(1-B^{s}\right)^{D} x_{t}=\theta(B) \Theta\left(B^{s}\right) \varepsilon_{t} \text {, for } t \in \mathbb{Z}
$$

where $s \in \mathbb{N}$ is the seasonal period, $B$ is the backward-shift operator, that is, $B^{s k} x_{t}=x_{t-s k},\left(1-B^{s}\right)^{D}$ is the seasonal difference operator, $\Phi(\cdot)$ and $\Theta(\cdot)$ are the polynomials of degrees $P$ and $Q$, respectively, defined by:

$$
\Phi\left(B^{s}\right)=\sum_{i=0}^{P}\left(-\Phi_{i}\right) B^{s i}, \Theta\left(B^{s}\right)=\sum_{j=0}^{Q}\left(-\Theta_{j}\right) B^{s j}
$$

where $\Phi_{i}, 1 \leq i \leq P$ and $\Theta_{j}, 1 \leq j \leq Q$ are constants and $\Phi_{0}=-1=\Theta_{0}$.

The seasonal difference operator $\left(1-B^{s}\right)^{D}$, with seasonality $s \in \mathbb{N}$, for all $D>-1$, is defined by means of the binomial expansion;

$$
\left(1-B^{s}\right)^{D}=\sum_{j=0}^{\infty}\left(\begin{array}{c}
D \\
j
\end{array}\right)\left(-B^{s}\right)^{j}
$$

where;

$$
\left(\begin{array}{c}
D \\
j
\end{array}\right)=\frac{\Gamma(1+D)}{\Gamma(1+j) \Gamma(1+D-j)}
$$

A compact form of Equation (1) and Equation (4) is given by:

$$
\phi(B) \Phi\left(B^{s}\right) \nabla^{d} x_{t}=\theta(B) \Theta\left(B^{s}\right) \varepsilon_{t} \text {, for } t \in \mathbb{Z}
$$


In Equation (8), the operator $\nabla^{d}$ is defined by

$$
\nabla^{d}=(1-B)^{d}\left(1-B^{s}\right)^{D}
$$

where $\boldsymbol{d}=(d, D) \in \mathbb{R}^{2}$ is the memory vector parameter, $d$ and $D$ are the fractionally parameters at non seasonal and seasonal frequencies, respectively. The fractional filters are:

$$
\left(1-B^{k}\right)^{l}=\sum_{j=0}^{\infty}\left(\begin{array}{l}
l \\
j
\end{array}\right)\left(-B^{k}\right)^{j}, \quad k=1, s \text { and } l=d, D
$$

where;

$$
\left(\begin{array}{l}
l \\
j
\end{array}\right)=\frac{\Gamma(1+l)}{\Gamma(1+j) \Gamma(1+l-j)}
$$

Suppose in Equation (8), $\phi(B) \Phi\left(B^{s}\right)=0$ and $\theta(B) \Theta\left(B^{s}\right)=0$ have no common zeros. Let also $|d+D|<0.5$ and $|D|<0.5$, the process $\left\{x_{t}\right\}_{t \in \mathbb{Z}}$ has spectral density function is given by;

$$
f_{x}(\lambda)=\frac{\sigma_{\varepsilon}^{2}}{2 \pi} \frac{\left|\theta\left(\mathrm{e}^{-i \lambda}\right)\right|^{2}\left|\Theta\left(\mathrm{e}^{-i \lambda s}\right)\right|^{2}}{\left|\phi\left(\mathrm{e}^{-i \lambda}\right)\right|^{2}\left|\Phi\left(\mathrm{e}^{-i \lambda s}\right)\right|^{2}}\left[2 \sin \left(\frac{\lambda}{2}\right)\right]^{-2 d}\left[2 \sin \left(\frac{\lambda s}{2}\right)\right]^{-2 D},
$$

where $0<\lambda \leq \pi$.

Note that according to [15]:

1) The processs $\left\{x_{t}\right\}_{t \in \mathbb{Z}}$ is stationary if $d+D<0.5, D<0.5$ and $\phi(B) \Phi\left(B^{s}\right) \neq 0$, for $|B| \leq 1$.

2) The stationary process $\left\{x_{t}\right\}_{t \in \mathbb{Z}}$ has a long memory property if $0<d+D<0.5, \quad 0<D<0.5$ and $\phi(B) \Phi\left(B^{s}\right) \neq 0$, for $|B| \leq 1$.

3) The stationary process $\left\{x_{t}\right\}_{t \in \mathbb{Z}}$ has an intermediate property if $-0.5<d+D<0, \quad-0.5<D<0$ and $\phi(B) \Phi\left(B^{s}\right) \neq 0$, for $|B| \leq 1$.

For convenience, we introduce the notation $\mathbb{Z}_{\geq}=\{k \in \mathbb{Z} \mid k \geq 0\}$, $\mathbb{Z}_{\leq}=\{k \in \mathbb{Z} \mid k \leq 0\}$ and $A=\{1, \cdots, s-1\} \subset \mathbb{N}$.

Theorem 2.1. Let $\left\{x_{t}\right\}_{t \in \mathbb{Z}}$ be the $\operatorname{SARFIMA}(0, d, 0)(0, D, 0) s$ process given by expression:

$$
(1-B)^{d}\left(1-B^{s}\right)^{D} x_{t}=\varepsilon_{t}
$$

with zero mean, $s \in \mathbb{N}$ as the seasonal period. Then the process $\left\{x_{t}\right\}_{t \in \mathbb{Z}}$ has autocovariance function of order $h, h \in \mathbb{Z}_{\geq}$, given by

$$
\gamma_{x}(h)= \begin{cases}\sigma_{\varepsilon}^{2} \sum_{v \in \mathbb{Z}_{\geq}} \gamma_{z}(s v) \gamma_{y}(h-s v), & \text { if } h=s l, l \in \mathbb{Z}_{\geq} ; \\ 0, & \text { if } h=s l+\zeta, \zeta \in A,\end{cases}
$$

where

1) The process $\left\{y_{t}\right\}_{t \in \mathbb{Z}}$ is an $\operatorname{ARFIMA}(0, d, 0)$ with autocovariance function of order $h, h \in \mathbb{Z}_{\geq}$, given by

$$
\gamma_{y}(h)=\frac{(-1)^{h} \Gamma(1-2 d)}{\Gamma(h-d+1) \Gamma(1-h-d)}
$$


2) The process $\left\{z_{t}\right\}_{t \in \mathbb{Z}}$ is an $\operatorname{SARFIMA}(0, D, 0)_{s}$ with autocovariance function of order $v, v \in \mathbb{Z}_{\geq}$, given by

$$
\gamma_{z}(s v+\xi)= \begin{cases}\frac{(-1)^{v} \Gamma(1-2 D)}{\Gamma(v-D+1) \Gamma(1-v-D)}=\gamma_{x}(v), & \text { if } \xi=0 \\ 0, & \text { if } \xi \in A\end{cases}
$$

For proof of Theorem (2.1) see [15] Theorem 2.1.

Theorem 2.2. Let $\left\{x_{t}\right\}_{t \in \mathbb{Z}}$ be a causal and invertible $\operatorname{SARFIMA}(p, d, q)(P, D, Q) s$ process given by the expression:

$$
\phi(B) \Phi\left(B^{s}\right)(1-B)^{d}\left(1-B^{s}\right)^{D} x_{t}=\theta(B) \Theta\left(B^{s}\right) \varepsilon_{t} \text { for } t \in \mathbb{Z}
$$

with zero mean, $s \in \mathbb{N}$ as the seasonal period. Suppose $\phi(B) \Phi\left(B^{s}\right)=0$ and $\theta(B) \Theta\left(B^{s}\right)=0$ have no common zeros. For $|d+D|<0.5,|D|<0.5$, then the process $\left\{x_{t}\right\}_{t \in \mathbb{Z}}$ has autocovariance function of order $h, h \in \mathbb{Z}_{\geq}$, given by

$$
\gamma_{x}(h)= \begin{cases}\sigma_{\varepsilon}^{2} \sum_{v \in \mathbb{Z}_{\geq}} \gamma_{z}(s v) \gamma_{y}(h-s v), & \text { if } h=s l, l \in \mathbb{Z}_{\geq} ; \\ 0, & \text { if } h=s l+\zeta, \zeta \in A,\end{cases}
$$

where

1) the process $\left\{y_{t}\right\}_{t \in \mathbb{Z}}$ is an $\operatorname{ARFIMA}(p, d, q)$ with autocovariance function given by $\gamma_{y}(\cdot)$.

2) the process $\left\{z_{t}\right\}_{t \in \mathbb{Z}}$ is an $\operatorname{SARFIMA}(P, D, Q)_{s}$ with autocovariance function given by $\gamma_{z}(\cdot)$.

For proof of Theorem (2.2) see [15] Theorem 2.2.

\subsection{The GARCH $(m, r)$ Model}

The $\operatorname{GARCH}(m, r)$ model can be obtained from Equation (1) by letting $\mathbb{E}\left[\varepsilon_{t} \mid F_{t-1}\right]=0$ and the conditional variance, $\mathbb{E}\left[\varepsilon_{t}^{2} \mid F_{t-1}\right]=h_{t}$ where $F_{t-1}$ is the $\sigma$ field generated by the past information $\left\{\varepsilon_{t-1}, \varepsilon_{t-2}, \cdots\right\}$. Let also $\varepsilon_{t} \mid F_{t-1} \sim N\left(0, h_{t}\right)$ and

$$
\varepsilon_{t}=z_{t} \sqrt{h_{t}}
$$

where $z_{t}$ is normal distributed with mean 0 and variance 1. [4] introduced the $\operatorname{GARCH}(m, r)$ model which defines the conditional variance equation as follows;

$$
h_{t}=\omega_{0}+\sum_{i=1}^{r} \alpha_{i} \varepsilon_{t-i}^{2}+\sum_{i=1}^{m} \beta_{i} h_{t-i}
$$

where $\omega_{0}>0, \alpha_{1}, \cdots, \alpha_{r}, \beta_{1}, \cdots, \beta_{m} \geq 0, r$ and $m$ are positive integer. Note that the GARCH model defined by (20) can be replaced by other conditional heteroscedastic models.

\subsection{The General SARFIMA-GARCH Model}

Let the $\operatorname{SARFIMA}(p, d, q)(P, D, Q) s-\operatorname{GARCH}(m, r)$ model be the discrete time series model of $\left\{y_{t}\right\}$ given by the following equation. 


$$
\begin{gathered}
y_{t}=\mu_{0}+\phi^{-1}(B) \Phi^{-1}\left(B^{s}\right)(1-B)^{-d}\left(1-B^{s}\right)^{-D} \theta(B) \Theta\left(B^{s}\right) \varepsilon_{t} \\
\varepsilon_{t}=z_{t} \sqrt{h_{t}}, \quad \varepsilon_{t} \mid F_{t-1} \sim N\left(0, h_{t}\right) \\
h_{t}=\omega_{0}+\sum_{i=1}^{r} \alpha_{i} \varepsilon_{t-i}^{2}+\sum_{i=1}^{m} \beta_{i} h_{t-i}
\end{gathered}
$$

\subsection{Variance of Variance in the Standard GARCH $(m, r)$ Model}

By rearranging the conditional variance Equation (20) for a $\operatorname{GARCH}(1,1)$ we obtain:

$$
\begin{aligned}
h_{t} & =\omega_{0}+\left(\alpha_{1}+\beta_{1}\right) h_{t-1}+\alpha_{1}\left(\varepsilon_{t-1}^{2}-h_{t-1}\right) \\
& =\omega_{0}+\gamma h_{t-1}+\alpha_{1} h_{t-1} \eta_{t-1}
\end{aligned}
$$

where $\gamma=\alpha_{1}+\beta_{1}$ and $\eta_{t}=z_{t}^{2}-1$. [16] have shown that the variance of variance is given by:

$$
\operatorname{Var}\left(h_{t}\right)=\alpha_{1}^{2} h_{t-1} \mathbb{E}\left[\eta_{t-1}\right]=\left(\kappa_{z}-1\right) \alpha_{1}^{2} h_{t-1}^{2}
$$

where $\kappa_{z}$ denotes the conditional kurtosis of $z_{t}$, which we assume to be finite constant. If the distribution of $z_{t}$ is standard normal, then $\kappa_{z}-1=2$.

[16] further rearranged the terms in Equation (22), the conditional variance equation becomes:

$$
\begin{aligned}
h_{t}-h_{t-1} & =\varphi\left(\tau-h_{t-1}\right)+\alpha_{1} \sqrt{h_{t-1}} \eta_{t-1} \\
& =\omega_{0}+\gamma h_{t-1}+\alpha_{1} \sqrt{h_{t-1}} \eta_{t-1}
\end{aligned}
$$

where $\varphi=1-\gamma$ determines the speed at which the conditional variance reverts to its long run mean $\tau$, that is, $\mathbb{E}(\tau)=\omega_{0}(1-\gamma)^{-1}$ and its corresponding variance becomes:

$$
\operatorname{Var}\left(h_{t}-h_{t-1}\right)=\left(\kappa_{z}-1\right) \alpha_{1}^{2} h_{t-1}
$$

\subsection{Intervention in ARIMA Models}

Traditional time series analysis has considered four types of interventions, see for instance, [17] [18] [19] and [20]. The four types of interventions are:

1) Additive Outlier (AO): represents an isolated spike.

2) Level Shift (LS): represents a step function.

3) Transitory or Temporary Change (TC): represents a spike that takes a few periods to disappear.

4) Innovative Outlier (IO): represents effects that depend on the ARIMA model for the observed series.

According to [21], the effect of an AO, a LS, or a TC on an observed series is independent of the ARIMA model whereas the effect of an IO on an observed series consist of an initial shock that propagates in the subsequent observations with the weights of the ARIMA model.

Let $y_{t}$ be a time series that can be described with the $\operatorname{SARIMA}(p, d, q)(P, D, Q) s$ model: 


$$
\phi(B) \Phi\left(B^{s}\right)(1-B)^{d}\left(1-B^{s}\right)^{D}\left(y_{t}-\mu_{0}\right)=\theta(B) \Theta\left(B^{s}\right) \varepsilon_{t} \text {, for } t=1, \cdots, n
$$

and let $x_{t}$ be the contaminated series containing $k$ outliers represented by:

$$
x_{t}=y_{t}+\sum_{j=1}^{k} \mu_{j} G_{j}(B) I_{t}^{\tau_{j}}
$$

where $\mu_{j}$ is the initial impact of the outlier at time $t=\tau_{j} ; I_{t}^{\tau_{j}}$ is an indicator variable such that it is 1 for $t=\tau_{j}$ and 0 otherwise; and $G_{j}(B)$ determines the dynamics of the intervention occurring at time $t=\tau_{j}$ according to the following schemes:

1) $\mathrm{AO}: G_{j}(B)=1$.

2) LS: $G_{j}(B)=(1-B)^{-1}$.

3) TC: $G_{j}(B)=(1-\delta B)^{-1}, 0<\delta<1$.

4) IO: $G_{j}(B)=\phi^{-1}(B) \Phi^{-1}\left(B^{s}\right)(1-B)^{-d}\left(1-B^{s}\right)^{-D} \theta(B) \Theta\left(B^{s}\right)$.

[21] came up with a new intervention type, Seasonal Level Shift (SLS), that can describe a perturbation mostly related to the seasonal component. Seasonal level shifts are the interventions that affect only certain quarters or months of a year. The SLS is a special kind of level shift that occurs in $\operatorname{SARIMA}(p, d, q)(P, D, Q) s$ at some point $t=\tau$ in time and reoccur regularly every year at same season say $s$ and its effect carries up to subsequent seasons. The basic model with SLS suggested by [21] is given by

$$
x_{t}=y_{t}+\mu G\left(B^{s}\right) I_{t}^{\tau} \text { where } G\left(B^{s}\right)=\left(1-B^{s}\right)^{-1}
$$

However $G\left(B^{s}\right)=\left(1-B^{s}\right)^{-1}$ causes an impact on trend which can only be removed by defining dynamic weights as

$$
G\left(B^{s}\right)=\frac{1}{1-B^{s}}-\frac{1}{s(1-B)}
$$

The dynamic impact was normalized as suggested by [22]:

$$
\begin{aligned}
G\left(B^{s}\right) & =\frac{s}{s-1}\left[\left(1-B^{s}\right)^{-1}-\frac{1}{s}(1-B)^{-1}\right] \\
& =\frac{s}{s-1}\left[\left(1+B^{s}+B^{2 s}+B^{3 s}+\cdots\right)-\frac{1}{s}\left(1+B+B^{2}+B^{3}+\cdots\right)\right] \\
& =\left[1-\frac{s}{s-1} B+\frac{s}{s-1} B^{s}\right]\left(1-B^{s}\right)^{-1}(1-B)^{-1}
\end{aligned}
$$

Thus model (28) becomes

$$
\begin{aligned}
x_{t} & =y_{t}+\mu G\left(B^{s}\right) I_{t}^{\tau} \\
& =y_{t}+\mu\left[1-\frac{s}{s-1} B+\frac{s}{s-1} B^{s}\right]\left(1-B^{s}\right)^{-1}(1-B)^{-1} I_{t}^{\tau}
\end{aligned}
$$

Equation (31) is the observed series indicating series that the occurrence of SLS affects the time series for several seasons at same quarters or month with magnitude of outlier $\mu$. The outlier is introduced in the model by generating a variable: 


$$
G\left(B^{s}\right) I_{t}^{\tau}= \begin{cases}0 & \text { for } t<\tau \\ 1 & \text { at } t=\tau+s_{j} \\ \frac{-1}{s-1} & \text { at } t=\tau+s_{j}+1, \tau+s_{j}+2, \cdots, \tau+s_{j}+s-1 \\ & j=1,2, \cdots\end{cases}
$$

However in this research, for a SLS intervention at period $t=\tau$, we shall stick to the simple specification model (28).

\section{SARFIMA-GARCH Models with Level Shift}

This section presents a natural extension of the SARFIMA-GARCH models to a case with level shift. We start with a standard shift in the mean, then a level shift in seasonality. We will also consider level shift in the volatility with its corresponding shift in seasonality.

\subsection{The SARFIMA Model with Level Shift}

The $\operatorname{SARFIMA}(p, d, q)(P, D, Q) s$ model is written as

$$
\phi(B) \Phi\left(B^{s}\right)(1-B)^{d}\left(1-B^{s}\right)^{D}\left(y_{t}-\mu_{0}\right)=\theta(B) \Theta\left(B^{s}\right) \varepsilon_{t} \text {, for } t=1, \cdots, n
$$

where $y_{t}$ is the time series at time $t, \mu_{0}$ is the unconditional mean of the process. We assume the noise process $\varepsilon_{t}$ to be Gaussian, with expectation zero and variance $\sigma_{\varepsilon}^{2}$.

To allow for a mean level shift, after time $t=\tau$ of the data, we write the sum of an unobserved SARFIMA process and the term for the mean level shift which we will denote as $\operatorname{LS}$-SARFIMA $(p, d, q)(P, D, Q) s$

$$
\begin{aligned}
x_{t} & =y_{t}+\mu_{11}(1-B)^{-1} I_{t}^{\tau} \\
& =y_{t}+\mu_{11} G_{11}(B) I_{t}^{\tau}
\end{aligned}
$$

where $x_{t}$ denotes the observed contaminated series; $y_{t}$ follows the SARFIMA process; $I_{t}^{\tau}$ is an indicator variable taking values 1 for $t=\tau$, and 0 otherwise. The parameter $\mu_{11}$ indicates the size of the mean level shift at time $t=\tau$; $G_{11}(B)$ determines the dynamics of the intervention occurring at time $t=\tau$. The mean level shift is an abrupt but permanent shift by $\mu_{11}$ in the series caused by an intervention.

The extension of (34) to $k$ level shifts is straightforward. We define $\mu_{1 j}$ as the $\tau_{j}^{\text {th }}$ shift in level, compared to the previous level, where $j=1, \cdots, k$. When we allow $k$ level changes at pre-specified time $t=\tau_{j}$, we can extend Equation (34) and Equation (35) to

$$
\begin{aligned}
x_{t} & =y_{t}+\sum_{j=1}^{k} \mu_{1 j}(1-B)^{-1} I_{t}^{\tau_{j}} \\
& =y_{t}+\sum_{j=1}^{k} \mu_{1 j} G_{1 j}(B) I_{t}^{\tau_{j}}
\end{aligned}
$$

The component $\sum_{j=1}^{k} \mu_{1 j}(1-B)^{-1} I_{t}^{\tau_{j}}$ allows the intercept of the SARFIMA 
model to fluctuate over time between $\mu_{0}$ and $\mu_{0}+\sum_{j=1}^{k} \mu_{1 j}$.

\subsection{The SARFIMA Model with Seasonal Level Shift}

Seasonal Level Shift in SARFIMA models denoted as SLS-SARFIMA type intervention display seasonal features in its pattern. Many economic series display breaks and anomalies within the previous restrictions. To illustrate the argument, the SLS is presented in Figure 1.

This paper covers the methodologically and computationally less complex approach by extending intervention detection procedures to cover shifts in the seasonal component in fractionally integrated SARFIMA models. The Seasonal Level Shift (SLS) intervention has an effect on the trend given by the step function of Figure 2(a), and an effect on the seasonal component shown in Figure 2(b).

A Seasonal Level Shift intervention at period $t=\tau$ would affect the series $y_{t}$ and is represented by model (38):

Illustration Seasonal Factors

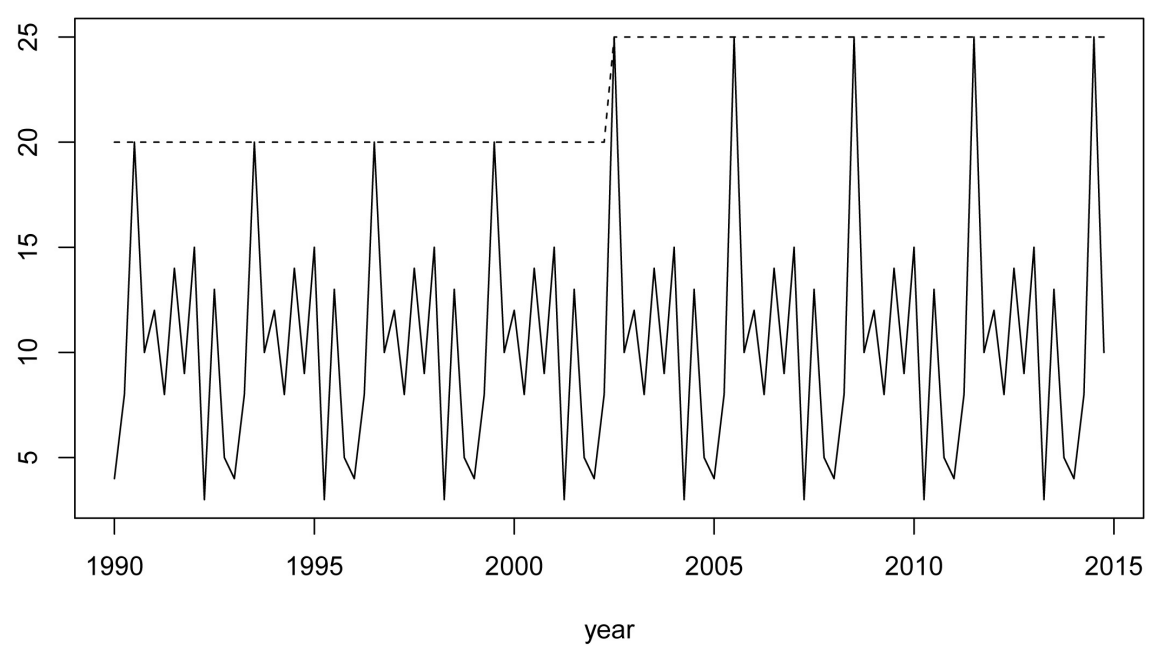

Figure 1. Seasonal level shift effect.

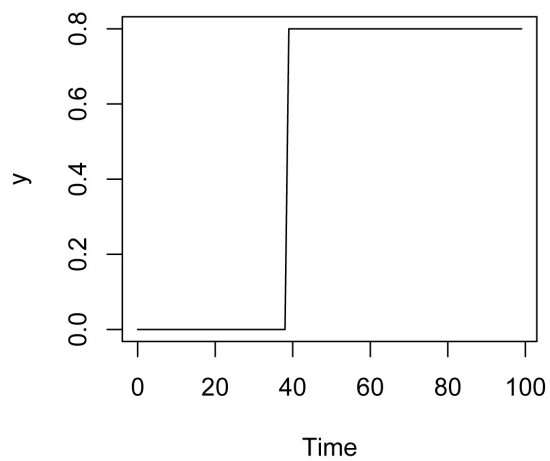

(a)

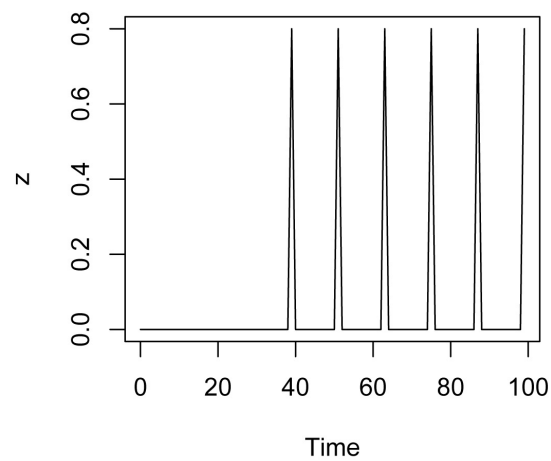

(b)

Figure 2. Seasonal level shift effect: (a) effects of a SLS on the trend cycle component. (b) effects of a SLS on the seasonal component. 


$$
x_{t}=\mu_{21} G_{21}\left(B^{s}\right) I_{t}^{\tau}+y_{t} \text { where } G_{21}\left(B^{s}\right)=\left(1-B^{s}\right)^{-1}
$$

Assuming the observed series contains $k$ seasonal level shifts at time $t=\tau_{j}$ for $j=1, \cdots, k$, their combined effect can be expressed in general form as:

$$
x_{t}=\sum_{j=1}^{k} \mu_{2 j} G_{2 j}\left(B^{s}\right) I_{t}^{\tau_{j}}+y_{t} \text { where } G_{2 j}\left(B^{s}\right)=\left(1-B^{s}\right)^{-1}
$$

The component $\sum_{j=1}^{k} \mu_{2 j}\left(1-B^{s}\right)^{-1} I_{t}^{\tau_{j}}$ allows the intercept of the SARFIMA model to fluctuate over time between $\mu_{0}$ and $\mu_{0}+\sum_{j=1}^{k} \mu_{2 j}$.

\subsection{The GARCH $(m, r)$ Model with Level Shift}

As indicated earlier, [4] introduced the $\operatorname{GARCH}(m, r)$ model which defines the conditional variance equation as follows;

$$
h_{t}=\omega_{0}+\sum_{i=1}^{r} \alpha_{i} \varepsilon_{t-i}^{2}+\sum_{i=1}^{m} \beta_{i} h_{t-i}
$$

To allow for a volatility level shift, denoted $\omega_{11}$, after time $t=\tau$ of the data, we write $h_{t}$ as the sum of an unobserved GARCH process and the term of the volatility level shift which we will denote as $\operatorname{LS}-\operatorname{GARCH}(m, r)$.

$$
\begin{aligned}
h_{t} & =\omega_{0}+\sum_{i=1}^{r} \alpha_{i} \varepsilon_{t-i}^{2}+\sum_{i=1}^{m} \beta_{i} h_{t-i}+\omega_{11}(1-B)^{-1} I_{t}^{\tau} \\
& =\omega_{0}+\sum_{i=1}^{r} \alpha_{i} \varepsilon_{t-i}^{2}+\sum_{i=1}^{m} \beta_{i} h_{t-i}+\omega_{11} G_{11}(B) I_{t}^{\tau}
\end{aligned}
$$

where $I_{t}^{\tau}$ is an indicator variable taking values 1 for $t=\tau$, and 0 otherwise. The parameter $\omega_{11}$ indicates the size of the volatility level shift at time $t=\tau$.

The extension of Equation (41) and Equation (42) to $k$ volatility level shifts is straightforward. We define $\omega_{1 j}$ as the $\tau_{j}^{\text {th }}$ shift in volatility level, compared to the previous level, where $j=1, \cdots, k$. When we allow $k$ volatility level changes at pre-specified time $t=\tau_{j}$, we can extend (41) and (42) to

$$
\begin{aligned}
h_{t} & =\omega_{0}+\sum_{i=1}^{r} \alpha_{i} \varepsilon_{t-i}^{2}+\sum_{i=1}^{m} \beta_{i} h_{t-i}+\sum_{j=1}^{k} \omega_{1 j}(1-B)^{-1} I_{t}^{\tau_{j}} \\
& =\omega_{0}+\sum_{i=1}^{r} \alpha_{i} \varepsilon_{t-i}^{2}+\sum_{i=1}^{m} \beta_{i} h_{t-i}+\sum_{j=1}^{k} \omega_{1 j} G_{1 j}(B) I_{t}^{\tau_{j}}
\end{aligned}
$$

The component $\sum_{j=1}^{k} \omega_{1 j}(1-B)^{-1} I_{t}^{\tau_{j}}$ governs the level shift movement of GARCH model intercept, that is baseline volatility, over time between $\omega_{0}$ and $\omega_{0}+\sum_{j=1}^{k} \omega_{1 j}$.

\subsection{The GARCH $(r, m)$ Model with Seasonal Level Shift}

Seasonal Level Shift in volatility denoted as SLS-GARCH type intervention display seasonal features in its pattern. The Seasonal Level Shift (SLS) intervention has an effect on the trend given by the step function and an effect on the seasonal component.

A Seasonal Level Shift intervention in GARCH model at period $t=\tau$ would 
affect the series $h_{t}$ and is represented by model (45) and (46):

$$
\begin{aligned}
h_{t} & =\omega_{0}+\sum_{i=1}^{r} \alpha_{i} \varepsilon_{t-i}^{2}+\sum_{i=1}^{m} \beta_{i} h_{t-i}+\omega_{21}\left(1-B^{s}\right)^{-1} I_{t}^{\tau} \\
& =\omega_{0}+\sum_{i=1}^{r} \alpha_{i} \varepsilon_{t-i}^{2}+\sum_{i=1}^{m} \beta_{i} h_{t-i}+\omega_{21} G_{21}\left(B^{s}\right) I_{t}^{\tau}
\end{aligned}
$$

Assuming the observed series contains $k$ seasonal level shifts at time $t=\tau_{j}$ for $j=1, \cdots, k$, their combined effect can be expressed in general form as:

$$
\begin{aligned}
h_{t} & =\omega_{0}+\sum_{i=1}^{r} \alpha_{i} \varepsilon_{t-i}^{2}+\sum_{i=1}^{m} \beta_{i} h_{t-i}+\sum_{j=1}^{k} \omega_{2 j}\left(1-B^{s}\right)^{-1} I_{t}^{\tau_{j}} \\
& =\omega_{0}+\sum_{i=1}^{r} \alpha_{i} \varepsilon_{t-i}^{2}+\sum_{i=1}^{m} \beta_{i} h_{t-i}+\sum_{j=1}^{k} \omega_{2 j} G_{2 j}\left(B^{s}\right) I_{t}^{\tau_{j}}
\end{aligned}
$$

The component $\sum_{j=1}^{k} \omega_{2 j}\left(1-B^{s}\right)^{-1} I_{t}^{\tau_{j}}$ allows the intercept of the GARCH model to fluctuate over time between $\omega_{0}$ and $\omega_{0}+\sum_{j=1}^{k} \omega_{2 j}$.

\subsection{The General SARFIMA-GARCH Model with Level Shift}

Extension of $y_{t}$ the $\operatorname{SARFIMA}(p, d, q)(P, D, Q) s-\operatorname{GARCH}(m, r)$ process to the case with standard level shift is given by the following equation which we will denote as LS-SARFIMA-LS-GARCH

$$
\begin{gathered}
x_{t}=y_{t}+\sum_{j=1}^{k} \mu_{1 j}(1-B)^{-1} I_{t}^{\tau_{j}} \\
\varepsilon_{t}=z_{t} \sqrt{h_{t}}, \quad \varepsilon_{t} \mid F_{t-1} \sim N\left(0, h_{t}\right) \\
h_{t}=\omega_{0}+\sum_{i=1}^{r} \alpha_{i} \varepsilon_{t-i}^{2}+\sum_{i=1}^{m} \beta_{i} h_{t-i}+\sum_{j=1}^{k} \omega_{1 j}(1-B)^{-1} I_{t}^{\tau_{j}}
\end{gathered}
$$

Similarly, extension of $y_{t}$ the $\operatorname{SARFIMA}(p, d, q)(P, D, Q) s-\operatorname{GARCH}(m, r)$ process to the case with seasonal level shift is given by the following equation which we will denote as SLS-SARFIMA-SLS-GARCH

$$
\begin{gathered}
x_{t}=y_{t}+\sum_{j=1}^{k} \mu_{2 j}\left(1-B^{s}\right)^{-1} I_{t}^{\tau_{j}} \\
\varepsilon_{t}=z_{t} \sqrt{h_{t}}, \quad \varepsilon_{t} \mid F_{t-1} \sim N\left(0, h_{t}\right) \\
h_{t}=\omega_{0}+\sum_{i=1}^{r} \alpha_{i} \varepsilon_{t-i}^{2}+\sum_{i=1}^{m} \beta_{i} h_{t-i}+\sum_{j=1}^{k} \omega_{2 j}\left(1-B^{s}\right)^{-1} I_{t}^{\tau_{j}}
\end{gathered}
$$

\section{Estimation of SLS-SARFIMA-SLS-GARCH Model Parameters}

\subsection{Estimation of SLS-SARFIMA Model Parameters}

The first step of estimation consists in estimating the $\operatorname{SARFIMA}(p, d, q)(P, D, Q) s$ assuming that the conditional variance is constant over time. By rearranging Equation (38) for one mean seasonal level shift we have:

$$
\phi(B) \Phi\left(B^{s}\right)(1-B)^{d}\left(1-B^{s}\right)^{D} y_{t}=\mu_{0}+\theta(B) \Theta\left(B^{s}\right) \varepsilon_{t}+\mu_{21}\left(1-B^{s}\right)^{-1} I_{t} .
$$


Therefore the null hypothesis of unconditional mean constancy becomes: $H_{0}: \mu_{21}=0$. Let $\psi_{1}=\left(d, D, \mu_{0}, \mu_{21}, \phi^{\prime}, \Phi^{\prime}, \theta^{\prime}, \Theta^{\prime}, \sigma_{\varepsilon}^{2}\right)^{\prime}$ be the approximate likelihood estimator (MLE) $\hat{\psi}_{1}$ of $\psi_{1}$ that maximizes the conditional log-likelihood:

$$
l_{t}\left(\psi_{1}\right)=-\frac{1}{2} \ln 2 \pi-\frac{1}{2} \ln \sigma_{\varepsilon}^{2}-\frac{1}{2} \frac{\varepsilon_{t}^{2}}{\sigma_{\varepsilon}^{2}} .
$$

The partial derivatives evaluated under $H_{0}$ are given by:

$$
\left.\frac{\partial l_{t}}{\partial \psi_{1}}\right|_{H_{0}}=-\left.\frac{\hat{\varepsilon}_{t}}{\sigma_{\varepsilon}^{2}} \frac{\partial \hat{\varepsilon}_{t}}{\partial \psi_{1}}\right|_{H_{0}}
$$

$\left.\frac{\partial \varepsilon_{t}}{\partial d}\right|_{H_{0}}=(1-B)^{d}\left(1-B^{s}\right)^{D} \hat{\phi}(B) \hat{\Phi}\left(B^{s}\right) \sum_{j}^{t-1} \frac{y_{t-j}}{j}+\sum_{j=1}^{Q} \hat{\Theta}_{j} \frac{\partial \hat{\varepsilon}_{t-j s}}{\partial d}$

$$
\begin{aligned}
& +\sum_{j=0}^{Q} \sum_{i=0}^{q} \hat{\Theta}_{j} \hat{\theta}_{i} \frac{\partial \hat{\varepsilon}_{t-i-j s}}{\partial d} \\
& \text { - }\left.\frac{\partial \varepsilon_{t}}{\partial D}\right|_{H_{0}}=(1-B)^{d}\left(1-B^{s}\right)^{D} \hat{\phi}(B) \hat{\Phi}\left(B^{s}\right) \sum_{j}^{t-1} \frac{y_{t-j s}}{j s}+\sum_{j=1}^{Q} \hat{\Theta}_{j} \frac{\partial \hat{\varepsilon}_{t-j s}}{\partial D} \\
& +\sum_{j=0}^{Q} \sum_{i=0}^{q} \hat{\Theta}_{j} \hat{\theta}_{i} \frac{\partial \hat{\varepsilon}_{t-i-j s}}{\partial D} \\
& \text { - }\left.\frac{\partial \varepsilon_{t}}{\partial \mu_{0}}\right|_{H_{0}}=-1+\sum_{j=1}^{Q} \hat{\Theta}_{j} \frac{\partial \hat{\varepsilon}_{t-j s}}{\partial \mu_{0}}+\sum_{j=0}^{Q} \sum_{i=0}^{q} \hat{\Theta}_{j} \hat{\theta}_{i} \frac{\partial \hat{\varepsilon}_{t-i-j s}}{\partial \mu_{0}} \\
& \text { - }\left.\frac{\partial \varepsilon_{t}}{\partial \mu_{21}}\right|_{H_{0}}=-\left(1-B^{s}\right)^{-1} I_{t}+\sum_{j=1}^{Q} \hat{\Theta}_{j} \frac{\partial \hat{\varepsilon}_{t-j s}}{\partial \mu_{21}}+\sum_{j=0}^{Q} \sum_{i=0}^{q} \hat{\Theta}_{j} \hat{\theta}_{i} \frac{\partial \hat{\varepsilon}_{t-i-j s}}{\partial \mu_{21}} \\
& \text { - }\left.\frac{\partial \varepsilon_{t}}{\partial \phi}\right|_{H_{0}}=\hat{\Phi}\left(B^{s}\right)(1-B)^{d}\left(1-B^{s}\right)^{D}\left(y_{t-1}, \cdots, y_{t-p}\right)+\sum_{j=1}^{Q} \hat{\Theta}_{j} \frac{\partial \hat{\varepsilon}_{t-j s}}{\partial \phi} \\
& +\sum_{j=1}^{Q} \sum_{i=0}^{q} \hat{\Theta}_{j} \hat{\theta}_{i} \frac{\partial \hat{\varepsilon}_{t-i-j s}}{\partial \phi} \\
& \left.\frac{\partial \varepsilon_{t}}{\partial \Phi}\right|_{H_{0}}=\hat{\phi}(B)(1-B)^{d}\left(1-B^{s}\right)^{D}\left(y_{t-s}, \cdots, y_{t-p s}\right)+\sum_{j=1}^{Q} \hat{\Theta}_{j} \frac{\partial \hat{\varepsilon}_{t-j s}}{\partial \Phi} \\
& +\sum_{j=0}^{Q} \sum_{i=0}^{q} \hat{\Theta}_{j} \hat{\theta}_{i} \frac{\partial \hat{\varepsilon}_{t-i-j s}}{\partial \Phi} \\
& \text { - }\left.\frac{\partial \varepsilon_{t}}{\partial \theta}\right|_{H_{0}}=\left(\sum_{j=0}^{Q} \hat{\Theta}_{j} \varepsilon_{t-1-j s}, \cdots, \sum_{j=0}^{Q} \hat{\Theta}_{j} \varepsilon_{t-q-j s}\right)+\sum_{j=1}^{Q} \hat{\Theta}_{j} \frac{\partial \hat{\varepsilon}_{t-j s}}{\partial \theta} \\
& +\sum_{j=1}^{Q} \sum_{i=0}^{q} \hat{\Theta}_{j} \hat{\theta}_{i} \frac{\partial \hat{\varepsilon}_{t-i-j s}}{\partial \theta} \\
& \left.\frac{\partial \varepsilon_{t}}{\partial \Theta}\right|_{H_{0}}=\left(\varepsilon_{t-s}, \varepsilon_{t-2 s}, \cdots, \varepsilon_{t-Q s}\right)+\left(\sum_{i=0}^{q} \hat{\theta}_{i} \varepsilon_{t-i-s}, \cdots, \sum_{i=0}^{q} \hat{\theta}_{i} \varepsilon_{t-i-Q s}\right) \\
& +\sum_{j=1}^{Q} \hat{\Theta}_{j} \frac{\partial \hat{\varepsilon}_{t-j s}}{\partial \Theta}+\sum_{j=0}^{Q} \sum_{i=0}^{q} \hat{\Theta}_{j} \hat{\theta}_{i} \frac{\partial \hat{\varepsilon}_{t-i-j s}}{\partial \Theta} \\
& \text { - }\left.\frac{\partial \varepsilon_{t}}{\partial \sigma_{\varepsilon}^{2}}\right|_{H_{0}}=-\frac{1}{2 \sigma_{\varepsilon}^{2}}+\frac{\hat{\varepsilon}_{t}^{2}}{2 \sigma_{\varepsilon}^{4}}
\end{aligned}
$$

Under $H_{0}$, the LM-type statistics is asymptotically distributed as $\chi^{2}$ with one degree of freedom: 


$$
L M_{1}=\left.\left.\frac{1}{2} \sum_{t=1}^{n} \frac{\partial l_{t}}{\partial \psi_{1}}\right|_{H_{0}}\left(\sum_{t=1}^{n}\left[\left.\frac{\partial \varepsilon_{t}}{\partial \psi_{1}}\right|_{H_{0}}\right]\left[\left.\frac{\partial \varepsilon_{t}}{\partial \psi_{1}}\right|_{H_{0}}\right]^{\prime}\right)^{-1} \sum_{t=1}^{n} \frac{\partial l_{t}}{\partial \psi_{1}}\right|_{H_{0}}
$$

\subsection{Estimation of SLS-GARCH Parameters}

Once the SLS-SARFIMA model is estimated and the residuals $\varepsilon_{t}$ are obtained, we test the alternative of SLS-GARCH specification with one volatility level shift against the null hypothesis of GARCH model. Let us rearrange model (41) with one volatility level shift:

$$
h_{t}=\omega_{0}+\alpha(B) \varepsilon_{t}+\beta(B) h_{t}+\omega_{21}\left(1-B^{s}\right)^{-1} I_{t}
$$

Therefore the null hypothesis of the unconditional variance constancy becomes: $H_{0}: \omega_{21}=0$. Let $\psi_{2}=\left(\omega_{0}, \omega_{21}, \alpha^{\prime}, \beta^{\prime}\right)^{\prime}$ be the vector of the SLS-GARCH model parameters and the quasi-likelihood function is given by:

$$
l_{t}\left(\psi_{2}\right)=-\frac{1}{2} \ln 2 \pi-\frac{1}{2} \ln h_{t}-\frac{1}{2} \frac{\varepsilon_{t}^{2}}{h_{t}} .
$$

The partial derivatives evaluated under $H_{0}$ are given by:

$$
\left.\frac{\partial l_{t}}{\partial \psi_{2}}\right|_{H_{0}}=\left.\frac{1}{2}\left[\frac{\hat{\varepsilon}_{t}^{2}}{\hat{h}_{0 t}}-1\right] \frac{\partial \ln h_{t}}{\partial \psi_{2}}\right|_{H_{0}}
$$

- $\left.\frac{\partial \ln h_{t}}{\partial \omega_{0}}\right|_{H_{0}}=\left(\hat{h}_{0 t}\right)^{-1}\left[1+\sum_{j=1}^{m} \hat{\beta}_{j} \frac{\partial \hat{h}_{t-j}}{\partial \omega_{0}}\right]$

- $\left.\frac{\partial \ln h_{t}}{\partial \omega_{21}}\right|_{H_{0}}=\left(\hat{h}_{0 t}\right)^{-1}\left[\left(1-B^{s}\right)^{-1} I_{t}+\sum_{j=1}^{m} \hat{\beta}_{j} \frac{\partial \hat{h}_{t-j}}{\partial \omega_{21}}\right]$

- $\left.\frac{\partial \ln h_{t}}{\partial \alpha}\right|_{H_{0}}=\left(\hat{h}_{0 t}\right)^{-1}\left[\left(\varepsilon_{t-1}^{2}, \cdots, \varepsilon_{t-r}\right)^{\prime}+\sum_{j=1}^{m} \hat{\beta}_{j} \frac{\partial \hat{h}_{t-j}}{\partial \alpha}\right]$

- $\left.\frac{\partial \ln h_{t}}{\partial \beta}\right|_{H_{0}}=\left(\hat{h}_{0 t}\right)^{-1}\left[\left(h_{t-1}, \cdots, h_{t-m}\right)^{\prime}+\sum_{j=1}^{m} \hat{\beta}_{j} \frac{\partial \hat{h}_{t-j}}{\partial \beta}\right]$

Under the null hypothesis, the "hats" indicates the maximum likelihood estimator and $\hat{h}_{0 t}$ denotes the conditional variance estimated at time $t$. Under $H_{0}$, the LM-type statistics is asymptotically distributed as $\chi^{2}$ with one degree of freedom:

$$
L M_{2}=\left.\left.\frac{1}{2} \sum_{t=1}^{n} \frac{\partial l_{t}}{\partial \psi_{2}}\right|_{H_{0}}\left(\sum_{t=1}^{n}\left[\left.\frac{\partial \ln h_{t}}{\partial \psi_{2}}\right|_{H_{0}}\right]\left[\left.\frac{\partial \ln h_{t}}{\partial \psi_{2}}\right|_{H_{0}}\right]^{\prime}\right)^{-1} \sum_{t=1}^{n} \frac{\partial l_{t}}{\partial \psi_{2}}\right|_{H_{0}}
$$

\section{Level Shift Detection and Estimation in SARFIMA-GARCH}

In this section we discuss how the iterative detective procedure described in [23] and [24] can be extended to allow for both detection and estimation of SLS in SARFIMA-GARCH intervention to be denoted SLS-SARFIMA and SLS-GARCH. 


\subsection{Detection and Estimation in SLS-SARFIMA}

Let $\psi_{1}=\left(d, D, \mu_{0}, \phi^{\prime}, \Phi^{\prime}, \theta^{\prime}, \Theta^{\prime}, \sigma_{\varepsilon}^{2}\right)^{\prime}$ be the vector of parameters in model (59)

$$
\phi(B) \Phi\left(B^{s}\right)(1-B)^{d}\left(1-B^{s}\right)^{D} y_{t}=\mu_{0}+\theta(B) \Theta\left(B^{s}\right) \varepsilon_{t}, t=1, \cdots, n
$$

and let us suppose, for the moment, that it is known. Further, suppose that the observed series is subject to the influence of a perturbation at time $t=\tau$ such that:

$$
x_{t}=\mu_{21} G\left(B^{s}\right) I_{t}^{\tau}+y_{t}
$$

where we assume that model (59) is stationary. Model (60) can be rewritten as a linear regression model presented in model (61) and model (62)

$$
x_{t}=\mu_{21} Z_{t}^{*}(\tau)+y_{t}
$$

where $Z_{t}^{*}(\tau)=G\left(B^{s}\right) I_{t}^{\tau}$ is an $n \times 1$. Writing model (61) in matrix form:

$$
\boldsymbol{x}=\mu_{21} \mathbf{Z}^{*}+\boldsymbol{y}
$$

where $\boldsymbol{x}=\left(x_{1}, \cdots, x_{n}\right)^{\prime} ; \boldsymbol{y}=\left(y_{1}, \cdots, y_{n}\right)^{\prime}$ and $\boldsymbol{Z}^{*}=\left(Z_{1}^{*}(\tau), \cdots, Z_{n}^{*}(\tau)\right)^{\prime}$.

According to [21], model (62) is a regression model with autocorrelation residuals and therefore the problem of estimating $\mu_{21}$ can be solved by Generalized Least Squares (GLS).

Let $\operatorname{Var}(\boldsymbol{x})=\sigma_{\varepsilon}^{2} \boldsymbol{\Sigma}$ with $\boldsymbol{\Sigma}$ a $n \times n$ matrix which depends on $\psi_{1}$ and which is assumed to be positive definite, and let $\Sigma=\boldsymbol{L}^{\prime} \boldsymbol{L}$ be the Cholesky decomposition of $\boldsymbol{\Sigma}$ with $\boldsymbol{L}$ lower triangular. Pre-multiplying Equation (62) by $\boldsymbol{L}^{-1}$, and setting $\boldsymbol{e}^{*}=\boldsymbol{L}^{-1} \boldsymbol{x}, \boldsymbol{Z}=\boldsymbol{L}^{-1} \boldsymbol{Z}^{*}$ and $\boldsymbol{e}=\boldsymbol{L}^{-1} \boldsymbol{y}$, we obtain the Ordinary Least Square (OLS) model

$$
\boldsymbol{e}^{*}=\mu_{21} \mathbf{Z}+\boldsymbol{e}
$$

where $\operatorname{Var}(\boldsymbol{e})=\sigma_{\varepsilon}^{2} \boldsymbol{I}_{n}$. The OLS estimator of $\mu_{21}$ and its variance are obtained from Equation (63) as

$$
\hat{\mu}_{21}=\left(\mathbf{Z}^{\prime} \mathbf{Z}\right)^{-1} \mathbf{Z}^{\prime} \boldsymbol{e}^{*} \text { and } \operatorname{Var}\left(\hat{\mu}_{21}\right)=\left(\mathbf{Z}^{\prime} \mathbf{Z}\right)^{-1} \sigma_{\varepsilon}^{2}
$$

As argued in [24], to move from the GLS model in (62) to the OLS model in (63), there is no need to evaluate the matrix $\Sigma$, since the application of the Kalman filter on the observed series $x$ yields the vector of standardized residuals $\boldsymbol{e}^{*}=\boldsymbol{L}^{-1} \boldsymbol{x}$. Similarly, the application of the same filter on vector $\boldsymbol{Z}^{*}$ provides the vector $\boldsymbol{Z}=\boldsymbol{L}^{-1} \boldsymbol{Z}^{*}$ from which Equation (64) can be computed.

To test the null hypothesis that the observation at time $t=\tau$ is not an intervention, one can use the standardized statistic.

$$
\lambda=\frac{\hat{\mu}_{21}}{\sqrt{\operatorname{Var}\left(\hat{\mu}_{21}\right)}}
$$

which, for known $\psi_{0}$, follows a standard normal distribution. By setting appropriate starting conditions (see, for example, ([24] [25], or [26]), the previous scheme extends in a straightforward manner to non-stationary series, for which $y_{t}$ follows model (59). 


\subsection{Mean Level Shift Detection in SARFIMA-GARCH}

The mean level shift detection test was previously derived by [27] for ARIMA models and extended by [20] for the fractionally integrated $\operatorname{ARFIMA}(p, d, q)$ models assuming conditional variance is constant over time. [28] extended the level shift detection test of the mean for a realization of time series $\left\{x_{t}\right\}$ satisfying LS-SARFIMA-LS-GARCH model. For our purpose a natural extension of the seasonal level shift detection test of the mean for a realization of time series $\left\{x_{t}\right\}$ satisfying SLS-SARFIMA and SLS-GARCH model was derived. In order to derive the test statistic, let us rewrite model (38), with only one mean seasonal level shift:

$$
x_{t}=y_{t}+\mu_{21}\left(1-B^{s}\right)^{-1} I_{t}^{\tau}
$$

The hypothesis to be tested is

$$
H_{0}: \mu_{21}=0 \text { against } H_{1}: \mu_{21} \neq 0
$$

which is based on $x_{1}, x_{2}, \cdots, x_{n}$ a realization of time series $\left\{x_{t}\right\}$ satisfying SARFIMA-GARCH model with mean seasonal level shift.

Model (66) can be rewritten as

$$
\left(1-B^{s}\right) x_{t}=\left(1-B^{s}\right) y_{t}+\mu_{21} I_{t}^{\tau}
$$

This implies transforming the series by differencing of $\left(1-B^{s}\right)$. Thus if $\mu_{21}=0,\left(1-B^{s}\right) x_{t}=\left(1-B^{s}\right) y_{t}$. The intervention parameter $\mu_{21}$ can be estimated using various methods like the maximum likelihood estimation and least square estimation. The least square estimate of $\mu_{21}$ if the mean intervention is at time $t=\tau$ is given by

$$
\hat{\mu}_{21}=\frac{\sum_{t=2}^{n}\left(1-B^{s}\right) x_{t} I_{t}^{\tau}}{\sum_{t=2}^{n} I_{t}^{2}}=\left(1-B^{s}\right) x_{\tau}=\left(1-B^{s}\right) y_{\tau}, \quad \tau=s+1, s+2, \cdots, n
$$

Extension of [27] test statistics can be written as:

$$
T_{n}=\max \left\{\left|T_{n}(t)\right|\right\}=\max \left\{\frac{\left|\hat{\mu}_{21}(t)\right|}{\sqrt{\operatorname{Var}\left[\hat{\mu}_{21}(t)\right]}}\right\}, t=s+1, s+2, \cdots, n
$$

where $\hat{\mu}_{21}(t)=x_{t}-x_{t-s}$ is the estimated intervention or impact at time $t=\tau$ and $\operatorname{Var}\left[\hat{\mu}_{21}(t)\right]$ is an estimate of the variance of $\hat{\mu}_{21}(t)$.

The distribution of the statistics is based on the fact that it is originally Normally distributed and then transformed to the Gamma distribution both of which belong to the Domain of Attraction of the Gumbel distribution with normalizing constants:

1) Normal Distribution:

$$
d_{n}=\sqrt{2 \ln (n)}-\frac{\ln (\ln (n))+\ln (4 \pi)}{2 \sqrt{2 \ln (n)}} \text { and } c_{n}=1 / \sqrt{2 \ln (n)}
$$

2) Gamma Distribution:

$$
d_{n}=2 \ln (n)-\ln (\ln (n))-2 \ln \Gamma(\pi) \text { and } c_{n}=2
$$


The maximum domain of attraction of the Gumbel is shown to some extent in [29] and in greater detail in [30].

Let the test statistics be given by

$$
S_{n}=\frac{T_{n}^{2}-d_{n}}{c_{n}}
$$

Then under $H_{0}: \mu_{21}=0$, the statistics $S_{n}$ satisfies

$$
S_{n} \stackrel{D}{\longrightarrow} F(y)=\exp \left(-\mathrm{e}^{-(y-\lambda) / \delta}\right), \text { as } n \rightarrow \infty
$$

where $D$ signifies convergence in distribution. Here, $\lambda \in R$ is location parameter and $\delta$ is scale parameter. The location parameter is also the mode of the distribution. Inverse of the $F(y)$ in Equation (73), is given by:

$$
y=\lambda-\delta \ln (-\ln (F))
$$

Thus a test of hypothesis can be conducted by comparing the test statistic $S_{n}$ in Equation (72) with an appropriate critical value. The largest $T_{n}^{2}(t)$ statistic is considered an intervention at the $\alpha$ significance if the $S_{n}$ value exceeds the critical value.

\subsection{Volatility Seasonal Level Shift Detection in SARFIMA-GARCH Model}

The second step is a natural extension of mean seasonal level shift detection in SARFIMA-GARCH model to volatility seasonal level shift detection in SARFMA-GARCH model. After estimating the SLS-SARFIMA model and the residuals $\varepsilon_{t}$ are obtained, we test, the alternative hypothesis of SLS-GARCH volatility level shift against the null hypothesis of GARCH model. Let us rewrite model (47) with one volatility seasonal level shift:

$$
h_{t}=g_{t}+\omega_{21}\left(1-B^{s}\right)^{-1} I_{t}^{\tau} \quad \text { where } g_{t}=\omega_{0}+\sum_{i=1}^{r} \alpha_{i} \varepsilon_{t-i}^{2}+\sum_{i=1}^{s} \beta_{i} h_{t-i}
$$

The hypothesis tested is

$$
H_{0}: \omega_{21}=0 \text { against } H_{1}: \omega_{21} \neq 0
$$

which is based on $h_{1}, h_{2}, \cdots, h_{n}$ a realization of time series $\left\{h_{t}\right\}$ from a GARCH model with seasonal level shift.

The derivation is based on the statistics

$$
\begin{aligned}
T_{n} & =\max \left\{\left|T_{n}(1)\right|, \cdots,\left|T_{n}(n)\right|\right\} \\
& =\max \left\{\frac{\left|\hat{\omega}_{21}(1)\right|}{\sqrt{\operatorname{Var}\left[\hat{\omega}_{21}(1)\right]}}, \cdots, \frac{\left|\hat{\omega}_{21}(n)\right|}{\sqrt{\operatorname{Var}\left[\hat{\omega}_{21}(n)\right]}}\right\}
\end{aligned}
$$

where $\hat{\omega}_{21}(t)=h_{t}-h_{t-s}$ is the estimated intervention or impact at time $t=\tau$ and $\operatorname{Var}\left[\hat{\omega}_{21}(t)\right]$ is an estimate of the variance of $\hat{\omega}_{21}(t)$.

Model (75) can be rewritten as

$$
\left(1-B^{s}\right) h_{t}=\left(1-B^{s}\right) g_{t}+\omega_{21} I_{t}^{\tau}
$$


Thus if $\omega_{21}=0,\left(1-B^{s}\right) h_{t}=\left(1-B^{s}\right) g_{t}$. The intervention parameter $\omega_{21}$ can be estimated using various methods like the maximum likelihood estimation and least square estimation. The least square estimate of $\omega_{21}$ if the volatility intervention is at time $t=\tau$ is

$$
\hat{\omega}_{21}=\frac{\sum_{t=2}^{n}\left(1-B^{s}\right) h_{t} I_{t}^{\tau}}{\sum_{t=2}^{n}\left(I_{t}^{\tau}\right)^{2}}=\left(1-B^{s}\right) h_{\tau}=\left(1-B^{s}\right) g_{\tau}, \quad \tau=s+1, s+2, \cdots, n
$$

The distribution of the statistics is based on the fact that it is originally Normally distributed and then transformed to the Gamma distribution both of which belong to the Domain of Attraction of the Gumbel distribution with normalizing constants:

1) Normal Distribution:

$$
d_{n}=\sqrt{2 \ln (n)}-\frac{\ln (\ln (n))+\ln (4 \pi)}{2 \sqrt{2 \ln (n)}} \text { and } c_{n}=1 / \sqrt{2 \ln (n)}
$$

2) Gamma Distribution:

$$
d_{n}=2 \ln (n)-\ln (\ln (n))-2 \ln \Gamma(\pi) \text { and } c_{n}=2
$$

The maximum domain of attraction of the Gumbel is shown to some extent in [29] and in greater detail in [30].

Let $\left\{h_{t}\right\}$ be a time series satisfying the volatility seasonal level shift model

$$
\left(1-B^{s}\right) h_{t}=\left(1-B^{s}\right) g_{t}+\omega_{21} I_{t}^{\tau}
$$

For any realization $h_{1}, h_{2}, \cdots, h_{n}$ of this time series, let the test statistics be given by:

$$
S_{n}=\frac{T_{n}^{2}-d_{n}}{c_{n}}
$$

Then under $H_{0}: \omega_{21}=0$, the statistics $S_{n}$ satisfies

$$
S_{n} \stackrel{D}{\longrightarrow} F(y)=\exp \left(-\mathrm{e}^{-(y-\lambda) / \delta}\right), \text { as } n \rightarrow \infty
$$

where $D$ signifies convergence in distribution. Thus a test of hypothesis can be conducted by comparing the test statistic $S_{n}$ Equation (83) with an appropriate critical value. The largest $T_{n}^{2}(i)$ statistic is considered as volatility intervention at the $\alpha$ level of significance if the test statistic $S_{n}$ value exceeds the critical value.

\subsection{Mean and Volatility Level Shift Detection in SARFIMA-GARCH}

Summary of the detection procedure is presented below:

1) Plot the data to get a picture of the type of series and possible seasonal level shift in the data.

2) Assume that the underlying SARFIMA-GARCH series $\left\{y_{t}\right\}$ contains no level shift and use maximum likelihood procedure to estimate its parameters.

3) The first test is performed to check the mean seasonal level shift which can be conducted as follows: 
a) State the hypothesis being tested, which is

$$
H_{0}: \mu_{21}=0 \text { against } H_{1}: \mu_{21} \neq 0
$$

b) Compute the residuals, the impact $\mu_{21}$ and the test statistics like the popular [27]'s likelihood ratio test statistics given by

$$
T_{n}=\max \left\{\left|T_{n}(t)\right|\right\}=\max \left\{\frac{\left|\hat{\mu}_{21}(t)\right|}{\sqrt{\operatorname{Var}\left[\hat{\mu}_{21}(t)\right]}}\right\}, t=s+1, s+2, \cdots, n
$$

Then compute the statistics:

$$
S_{n}=\frac{T_{n}^{2}-d_{n}}{c_{n}}
$$

c) Determine the critical values to use in the test.

d) Determine whether observations are seasonal level shifts and remove each from the series by subtracting the value of the impact $\mu_{2 i}, i=1, \cdots, k$ then apply the SARFIMA-GARCH modeling procedure to obtain the adequate model.

4) The second test is performed to check the volatility level shift which can be conducted as follows:

a) State the hypothesis being tested, which is

$$
H_{0}: \omega_{21}=0 \text { against } H_{1}: \omega_{21} \neq 0
$$

b) Compute the residuals, the impact $\omega_{21}$ and the test statistics like the popular [27]'s likelihood ratio test statistics given by

$$
\begin{aligned}
T_{n} & =\max \left\{\left|T_{n}(1)\right|, \cdots,\left|T_{n}(n)\right|\right\} \\
& =\max \left\{\frac{\left|\hat{\omega}_{21}(1)\right|}{\sqrt{\operatorname{Var}\left[\hat{\omega}_{21}(1)\right]}}, \cdots, \frac{\left|\hat{\omega}_{21}(n)\right|}{\sqrt{\operatorname{Var}\left[\left|\hat{\omega}_{21}(n)\right|\right]}}\right\}
\end{aligned}
$$

Then compute the statistics:

$$
S_{n}=\frac{T_{n}^{2}-d_{n}}{c_{n}}
$$

c) Determine the critical values to use in the test.

d) Determine whether observations are level shifts and remove each from the series by subtracting the value of the impact $\omega_{2 i},[i=1, \cdots, k]$ then apply the SARFIMA-GARCH modeling procedure to obtain the adequate model.

\section{Conclusions}

This paper focused on the theoretical derivation of the class of seasonal fractionally integrated autoregressive moving average-conditional heteroskedastisticty (SARFIMA-GARCH) models, with level shift type intervention. The following derivations were established:

1) A natural extension of the seasonal level shift detection test of the mean for a time series satisfies SLS-SARFIMA.

2) A natural extension of the seasonal level shift detection test of the volatility 
for a time series satisfies SLS-GARCH.

3) Test statistics that are useful to examine if seasonal level shift in an SARFIMA-GARCH model was established.

4) Estimation of SLS-SARFIMA and SLS-GARCH parameters was derived using quasi maximum likelihood estimation.

To appreciate the procedure, we derived a simulation study consisting of simulation of critical values for mean and volatility seasonal level shift, simulating different sizes of mean and volatility seasonal level shift impact, performing detection test and conducting the power of the mean level shift detection procedure which are considered in a separate paper by the same authors.

\section{Conflicts of Interest}

The authors declare no conflicts of interest regarding the publication of this paper.

\section{References}

[1] Granger, C.W. and Joyeux, R. (1980) An Introduction to Long-Memory Time Series Models and Fractional Differencing. Journal of Time Series Analysis, 1, 15-29. https://doi.org/10.1111/j.1467-9892.1980.tb00297.x

[2] Hosking, J.R. (1981) Fractional Differencing. Biometrika, 68, 165-176. https://doi.org/10.1093/biomet/68.1.165

[3] Robinson, P.M. and Zaffaroni, P. (1998) Nonlinear Time Series with Long Memory: A Model for Stochastic Volatility. Journal of Statistical Planning and Inference, 68, 359-371. https://doi.org/10.1016/S0378-3758(97)00149-3

[4] Bollerslev, T. (1986) Generalized Autoregressive Conditional Heteroskedasticity. Journal of Econometrics, 31, 307-327. https://doi.org/10.1016/0304-4076(86)90063-1

[5] Bollerslev, T., Engle, R.F. and Wooldridge, J.M. (1988) A Capital Asset Pricing Model with Time-Varying Covariances. Journal of Political Economy, 96, 116-131. https://doi.org/10.1086/261527

[6] Weiss, A.A. (1984) ARMA Models with Arch Errors. Journal of Time Series Analysis, 5, 129-143. https://doi.org/10.1111/j.1467-9892.1984.tb00382.x

[7] Ling, S.Q. and Li, W.K. (1997) On Fractionally Integrated Autoregressive Moving-Average Time Series Models with Conditional Heteroscedasticity. Journal of the American Statistical Association, 92, 1184-1194. https://doi.org/10.1111/j.1467-9892.1984.tb00382.x

[8] Reisen, V.A., Sarnaglia, A.J.Q., Reis Jr., N.C., L’Evy-Leduc, C. and Santos, J.M. (2014) Modeling and Forecasting Daily Average PM10 Concentrations by a Seasonal Long-Memory Model with Volatility. Environmental Modelling \& Software, 51, 286-295. https://doi.org/10.1016/j.envsoft.2013.09.027

[9] Tong, H. (2011) Nonlinear Time Series Analysis.

[10] Narayan, P.K., Liu, R. and Westerlund, J. (2016) A Garch Model for Testing Market Efficiency. Journal of International Financial Markets, Institutions and Money, 41, 121-138.

[11] Baillie, R.T., Chung, C.-F. and Tieslau, M.A. (1996) Analysing Inflation by the Fractionally Integrated Arfima-Garch Model. Journal of Applied Econometrics, 11, 
23-40.

https://doi.org/10.1002/(SICI)1099-1255(199601)11:1\%3C23::AID-JAE374\%3E3.0.C $\underline{\mathrm{O} ; 2-\mathrm{M}}$

[12] Montanari, A., Rosso, R. and Taqqu, M.S. (2000) A Seasonal Fractional ARIMA Model Applied to the Nile River Monthly Flows at Aswan. Water Resources Research, 36, 1249-1259. https://doi.org/10.1029/2000WR900012

[13] Giraitis, L. and Leipus, R. (1995) A Generalized Fractionally Differencing Approach in Long-Memory Modeling. Lithuanian Mathematical Journal, 35, 53-65. https://doi.org/10.1007/BF02337754

[14] Box, G.E., Jenkins, G.M., Reinsel, G.C. and Ljung, G.M. (2015) Time Series Analysis: Forecasting and Control. John Wiley \& Sons, Hoboken.

[15] Bisognin, C. and Lopes, S.R.C. (2009) Properties of Seasonal Long Memory Processes. Mathematical and Computer Modelling, 49, 1837-1851. https://doi.org/10.1016/j.mcm.2008.12.003

[16] Ishida, I. and Engle, R.F. (2002) Modeling Variance of Variance: the Square Root, the Affine, and the Cev Garch Models. Dept. Finances, New York.

[17] Fox, A.J. (1972) Outliers in Time Series. Journal of the Royal Statistical Society: Series $B$ (Methodological), 34, 350-363.

https://doi.org/10.1111/j.2517-6161.1972.tb00912.x

[18] Tsay, R.S. (1986) Time Series Model Specification in the Presence of Outliers. Journal of the American Statistical Association, 81, 132-141.

https://doi.org/10.1080/01621459.1986.10478250

[19] Chen, C. and Liu, L.-M. (1993) Joint Estimation of Model Parameters and Outlier Effects in Time Series. Journal of the American Statistical Association, 88, 284-297. https://doi.org/10.1080/01621459.1993.10594321

[20] Chareka, P., Matarise, F. and Turner, R. (2006) A Test for Additive Outliers Applicable to Long Memory Time Series. Journal of Economic Dynamics and Control, 30, 595-621. https://doi.org/10.1016/j.jedc.2005.01.003

[21] Kaiser, R. and Maravall Herrero, A. (1999) Seasonal Outliers in Time Series. Banco de Espana, Documentos de trabajo No. 9915, Madrid.

[22] Palate, J. (2006) Reusable Components for Seasonal Adjustment: A New Implementation of Tramo-Seats. Proceedings of the Conference on Seasonality, Seasonal Adjustment and Their Implications for Short-Term Analysis and Forecasting, Eurostat, Luxembourg, 1-25.

http://ec.europa.eu/eurostat/documents/4578629/4579724/PALATE-FINAL.pdf

[23] Tsay, R. (2005) Analysis of Financial Time Series. Vol. 543, Financial Econometrics, John Wiley \& Sons, Hoboken, New Jersey.

[24] G'Omez, V. and Maravall, A. (1994) Estimation, Prediction, and Interpolation for Nonstationary Series with the Kalman Filter. Journal of the American Statistical Association, 89, 611-624. https://doi.org/10.1080/01621459.1994.10476786

[25] Kohn, R. and Ansley, C.F. (1985) Efficient Estimation and Prediction in Time Series Regression Models. Biometrika, 72, 694-697.

https://doi.org/10.1093/biomet/72.3.694

[26] Bell, W. and Hillmer, S. (1991) Initializing the Kalman Filter for Nonstationary Time Series Models. Journal of Time Series Analysis, 12, 283-300. https://doi.org/10.1111/j.1467-9892.1991.tb00084.x

[27] Chang, I., Tiao, G.C. and Chen, C. (1988) Estimation of Time Series Parameters in the Presence of Outliers. Technometrics, 30, 193-204. 
https://doi.org/10.1080/00401706.1988.10488367

[28] Dhliwayo, L., Matarise, F. and Chimedza, C. (2020) Autoregressive Fractionally Integrated Moving Average-Generalized Autoregressive Conditional Heteroskedasticity Model with Level Shift Intervention. Open Journal of Statistics, 10, 341-362.

https://doi.org/10.4236/ojs.2020.102023

[29] Leadbetter, M.R., Lindgren, G. and Rootzen, H. (1983) Extreme and Related Properties of Random Sequence and Processes. Springer-Verlag, New York.

[30] Embrechts, P., Klppelberg, C. and Mikosch, T. (1997) Modeling Extremal Events for Insurance and Finance. Springer-Verlag, Berlin.

https://doi.org/10.1007/978-3-642-33483-2 